\title{
Binary Zero-Power Sensors: an alternative solution for power-free energy-autonomous sensor systems
}

\author{
Thomas Frank • Gerald Gerlach • Arndt Steinke
}

Received: 16 August 2011/ Accepted: 26 May 2012/Published online: 15 June 2012

(C) The Author(s) 2012. This article is published with open access at Springerlink.com

\begin{abstract}
Energy-autarkic sensor systems use energy from their environment for operation and data transfer. This needs both an efficient energy harvesting strategy and an appropriate energy storage management but also ultralow-power technologies for sensors, signal processing, and wireless signal transfer. Binary Zero-Power Sensors are threshold switches which take the switching energy directly from the quantity to be measured. For this purpose stimuli-responsive polymers—in particular hydrogels—are used which swell upon the influence of particular quantities like humidity, temperature or $\mathrm{pH}$ value. The swelling causes the deflection of a flexure element closing or opening the contacts of a switch. In this manner they deliver the switching states "ON" and "OFF" without any additional electrical energy.
\end{abstract}

\section{Introduction}

Sensors allow the monitoring of our surrounding environment and its conditions enabling us to control many aspects of our life. This concerns housing, mobility (car), energy consumption, environment protection and many other fields. Sensor industry is a very fast-growing industry with an average growth rate of more than $8 \%$ p.a. during the last 9 years (AMA 2012). Interestingly, it is estimated that

T. Frank $(\bowtie) \cdot$ A. Steinke

CiS Forschungsinstitut für Mikrosensorik und Photovoltaik

GmbH, Konrad-Zuse-Straße 14, 99099 Erfurt, Germany

e-mail: tfrank@cismst.de

G. Gerlach

Solid-State Electronics Laboratory, Electrical and Computer Engineering Department, Technische Universität Dresden, 01062 Dresden, Germany more than $70 \%$ of all sensors are used as threshold sensors (binary sensors) detecting just a certain value of a measuring quantity where a particular action will be triggered. Usually, technical solutions comprise a sensor element and a following relay for starting the targeted action.

Triggered by the unprecedented opportunities of micro electro mechanical systems (MEMS) technology in the 1970s (Petersen 1982) a lot of activities were started to integrate sensor technology and electronic relays to achieve binary sensors. In 1979 Petersen described micromechanical membrane switches as a new class of electronic devices (Petersen 1979). These switches had operating characteristics between conventional silicon transistors and mechanical electromagnetic relays. They could be manufactured by batch fabrication on silicon wafers using conventional semiconductor techniques like photolithography. The devices had dimensions in the range of less than $100 \mu \mathrm{m}$ showing the enormous potential of miniaturization for relays. However, these relays were controlled electrostatically requiring permanent electrical energy supply.

In the following years and decades many approaches have been introduced targeting miniaturization, energy consumption, and physical principles to control the switch. Beside electrostatically controlled relays (Drake et al. 1995) other control principles have been proposed, e.g., thermal bimorph solutions (Seki et al. 1997; Qui et al. 2003). Hei et al. proposed a MEMS-based relay for ultralow-power digital logic applications. Their switching behavior was improved in such a way that the supply voltage could be scaled down further than for CMOS devices (Hei et al. 2009). Prototype relays are also fabricated using CMOS-compatible processes. In the meanwhile MEMS micro relays have entered the mass market, e.g., for RF switches and optical switches with improved optical and mechanical performance (Suzuki 2002; 
Eloy 2006). Nevertheless, all these approaches need electrical energy to maintain their sensor function. The lion's share of energy is required for wireless data transmission but the power consumption of micro-controllers and sensors is also not negligible. Solutions using batteries are sufficient for systems consisting of micro-controllers, sensors and data transmission units, in which the latter two are turned on after reaching a given threshold value. An alternative could be energy harvesting devices (Beeby et al. 2006; CookChennault et al. 2008) which increase system complexity, size and prize. There are various energy management scenarios known which minimize fuel consumption. A prominent example is the "power-down" operating condition of electrical circuits. A further significant reduction in the required electrical power could be achieved, if the current consumption of the sensors is reduced to zero, even in case they are online.

In this work we describe a novel kind of binary sensors is described which does not need electrical energy for the switching function of any kind of measurand (Rangelow et al. 2009). Such Binary Zero-Power Sensors (BIZEPS) are threshold switches which take the switching energy directly from the quantity to be measured. For this purpose stimuli-responsive polymers-in particular hydrogels-are used which swell upon the influence of particular quantities like pressure, humidity, temperature, $\mathrm{pH}$ value, concentrations of chemical species in aqueous solutions or others. The swelling causes the deflection of a flexure element closing or opening the contacts of a switch as it is normally used in conventional relays.

The outstanding advantage of BIZEPS over other solutions is the elimination of costly battery solutions or energy harvesting measures. In contrast to these, the BIZEPS themselves need no external power supply and are thus literally energy-independent.

In the following we will describe the general set-up of a BIZEPS sensor, will discuss possible stimuli-responsive swelling materials - in particular polymers and hydrogels, respectively-and will explain first experiments using pressure sensor chips as the flexure element for the switching transducer element.

\section{Construction of the Binary Zero-Power Sensors}

Figure 1 shows a state-of-the-art autarkic system where the analog sensor needs continuous power supply. In contrast to this, systems using sensors based on the BIZEPS-concept can remain in the power-down state even though the binary sensor is online (Fig. 2). Thus, the consumption of electrical power is zero or merely in the order of $30 \mu \mathrm{W}$. On the occurrence of a well-defined event, the system is activated by the Binary-Zero-Power-Sensor and, hence,

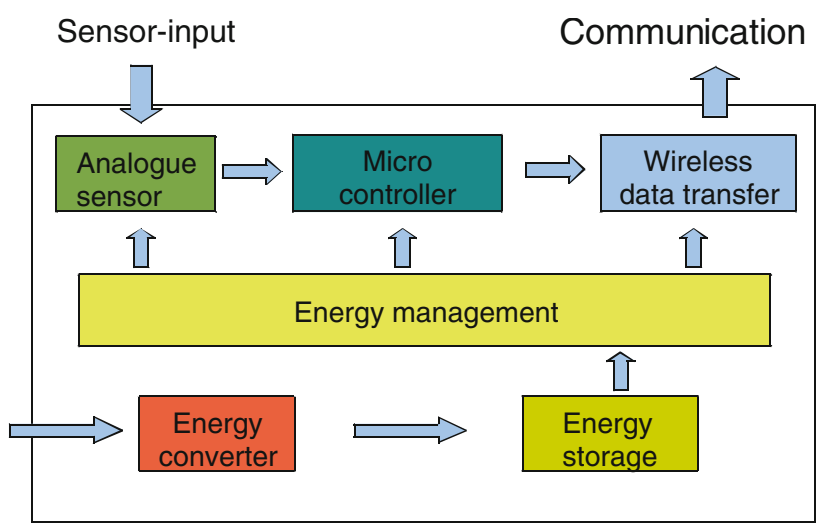

Fig. 1 Autonomous, networked sensor systems: state of the art

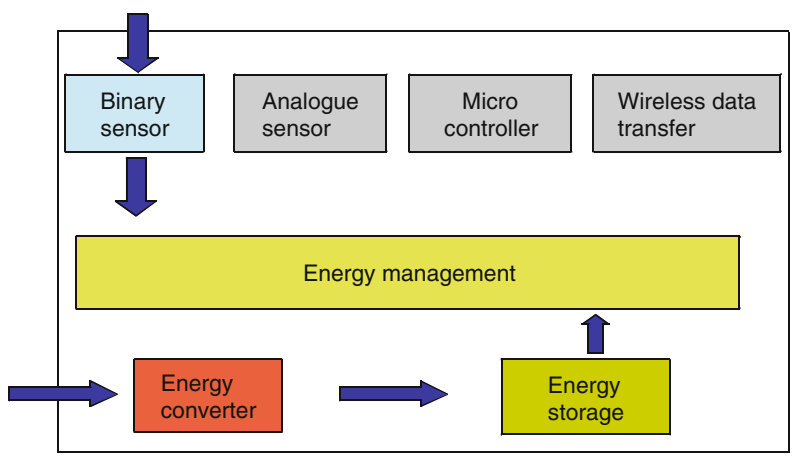

(a)

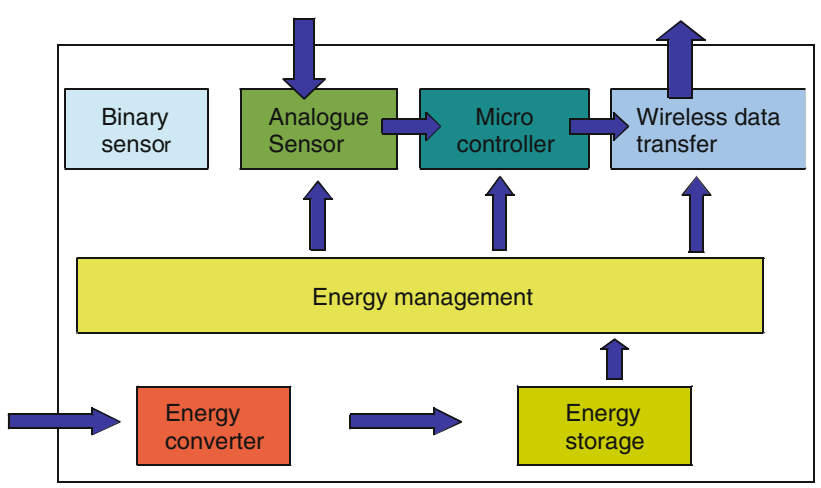

(b)

Fig. 2 Binary-Zero-Power Sensor as autonomous networked sensor systems with signalling system, a power down, b power on

powered on. Figure 3 shows the basic structural design of such a power-free binary sensor together with its essential components. The measurand affects a sensitive material, which swells or shrinks under the measurand's influence. This volume change is converted by the transducer into a switching movement as well as a contact force. The generated mechanical deflection closes an electrical contact, depending on the value of the measurand. 


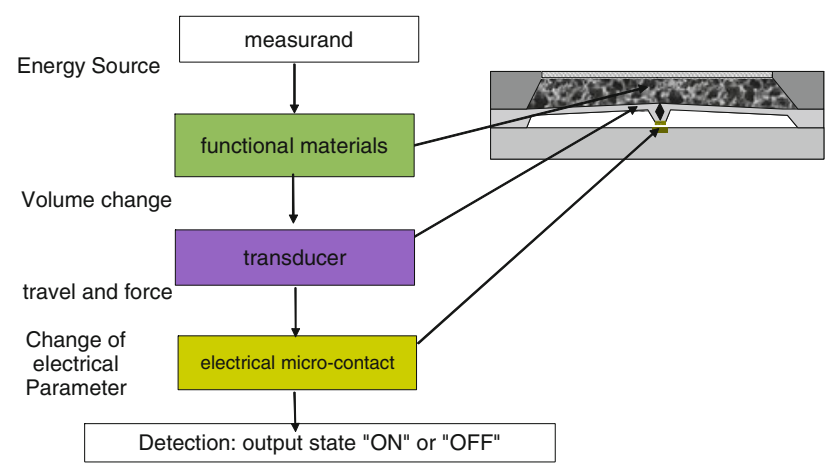

Fig. 3 Function principle and components of Binary Zero-Power Sensors

In this way, power-free sensors directly use the energy of the measured variable (ambient energy) for the switching of the electrical contacts, a multi-stage energy conversion is unnecessary. For the actual switching procedure, no electrical power supply is required, thus no cable losses occur. External electrical power is required only for the actions targeted by the switching, e.g., switching on certain functions.

Heart of this new type of sensor is a stimuli-responsive functional material, which swells as a function of the stimulus, such as temperature, pressure, humidity or $\mathrm{pH}$. The movement is achieved due to the coupling of the sensitive functional materials to the transducer. Cantilevers and membranes (bending plates) are the most common elements to transform the material's volume change into the mechanical movement. The generated displacement directly closes or opens the contacts of a built-in electrical switch.

Materials which show a specific swelling can be used as sensitive functional material. For instance, bimetal thermometers take advantage of the different thermal expansion of different metals. The resulting deflection of the appropriate bimetallic strip is proportional to the product of temperature change and the difference in thermal expansion coefficients of the two metals.

\section{Stimuli-sensitive swelling materials}

Of particular interest for this novel kind of binary sensors are stimuli-responsive materials, which swell or shrink as function of corresponding stimuli like temperature, pressure or humidity. The combination of the stimuli-responsive material with a mechanical micro switch avoids separate electrical function components for the switching operation. However, calibration and error correction have to be realized mechanically in the system material/deflectable mechanical element/micro switch instead by the usage of electrical measures.
The field of application of such zero-power sensors depends on the swelling behavior of the swellable material. The spectrum of available materials is very diverse ranging from the thermal extension of materials for temperature threshold switches via hygroscopic and, hence, humiditydependent materials to hydrogels which show swelling behavior in aqueous solutions with respect to the concentration of particular ions (e.g., $\mathrm{H}^{+}$-ions, i.e. $\mathrm{pH}$ value) or other chemical species.

\subsection{Temperature extension}

Bimetal thermometers use the difference in the thermal extension of different metals. The resulting deflection of a bimetal is proportional to the product of temperature change $\Delta T$ and the difference of the coefficients $\alpha_{1, T}$ of the thermal extension of the contributing metals. The largest deflection of such bimetallic elements occurs at the free end of a one-side clamped cantilever. If two-side clamped cantilevers or fixed clamped flexure plates are used thenfor symmetry reasons-one of the layers must not cover the other layer entirely. In the other case no deflection would occur because only in-plane stresses would evolve whereas the bending moment would be balanced by the clamping preventing the bimorph's deflection.

As an example, Seki et al. proposed a thin Si clampedclamped beam which is deformed by buckling due to thermal $\mathrm{SiO}_{2}$ built-in stress and thermal stress (Seki et al. 1997). The actuator was fabricated by a micromachining technology using sealed cavities. Its size is 2.5 (length) $\times$ 0.5 (width) $\times 0.02 \mathrm{~mm}$ (thickness). The deflection at the center point amounted to $25 \mu \mathrm{m}$. Qui et al. also reported the design, fabrication and testing of a thermally actuated bistable MEMS relay (Qui et al. 2003). Here, mechanical bistability ensures zero actuation power in both the on- and the off-states, and permits actuation with a transient thermal actuator. In the off-state, this relay stands off more than $200 \mathrm{~V}$, in the on-state, it exhibits a minimum total resistance of $60 \mathrm{n} \Omega$.

\subsection{Humidity extension}

Humidity-sensitive deformations can be excited in a very similar way to bimetals if a humidity-absorbing layer is deposited on an elastic carrier material. The humidityinduced swelling of the humidity-absorbing layer (e.g., polymers) leads to the bending of the bi-layer compound. As for bimetals, reliable and long-term stable adhesion between the polymer and the substrate is required. Promising polymers for such humidity-sensitive sensors are in particular polyimide (PI) (Gattiglia and Russel (1989), polyester (PE) (Ouchi et al. 1992) and polyethersulfone (PES). Their swelling behavior due to relative humidity has 
been studied intensively with respect to high sensitivity and sufficient long-term sensitivity (Buchhold et al. 1998a, b; Buchhold and Gerlach 2000). Modification of pore size (free volume between the polymer chains) and pore density within the polymers can lead to

- a higher uptake of water molecules and, hence, to a larger sensitivity,

- an improvement of the selectivity with respect to other gas molecules, and

- shorter response times.

Studies have shown that ion beam modification can enhance sensitivity up to one order of magnitude (Guenther et al. 2001; Guenther 2009).

\subsection{Stimulus-responsive swelling of hydrogels}

Another promising group of swellable polymers are hydrogels (Gerlach and Arndt 2009). Their molecule chains are connected chemically or physically to a two- or three-dimensional network. In the presence of appropriate solvents the network starts to swell due to the uptake of the solvent. The swollen network is called gel or-in the case of aqueous solutions as solvent-hydrogel. Hydrogel swelling or shrinking leads to a conversion from chemical into mechanical energy and vice versa generating enormous forces. This behavior enables the usage of hydrogels for sensors and actuators (Gerlach et al. 2004, 2005; Guenther et al. 2005; Arndt et al. 2000). It can be used favorably for BIZEPS sensors considered here.

The function principle of hydrogels can be exemplarily explained for $\mathrm{pH}$-sensitive compounds of polyvinyl alcohol (PVA) und polyacryl acid (PAA). The PVA provides the stable scaffold of the gel whereas the PAA acts as the sensitive component and, hence, is responsible for the polyelectrolytic character of the gel. The PVA/PAA compound has to be conditioned after crosslinking of the polymer network. This can be done in deionized water. Water molecules coupled to the PAS via hydrogen bonds can leave the hydrogel if the carboxyl group $(-\mathrm{COOH})$ of the PAA dissociates electrolytically. Water molecules will split into protons $\left(\mathrm{H}^{+}\right)$ and carboxylate ions (COO-) leading to a repulsion of the carboxylate ions and, hence, to swelling. Any change of the properties of the aqueous solution will change the equilibrium of the system of gel and chemical species. The reaction time depends on the temporal behavior of the corresponding processes:

- The basic and acidic components of the aqueous solution have to diffuse into the gel initiating swelling and shrinking, respectively.

- Water has to penetrate or to leave the gel.
- Other components of the fluid have to diffuse into or out of the gel depending on the concentration gradient.

All of these processes occur simultaneously. The optimum gel thickness should be a compromise between high sensitivity (large thickness) and short response time (small thickness) (Thong 2006). Porous gels can lead to a much faster response time of such sensors.

The selection of the appropriate hydrogel depends on the quantity to be measured, e.g., temperature, pressure, humidity, but also chemical or even biological quantities [Guenther et al. 2005; Guenther 2009; Gerlach et al. 2005; Gerlach and Arndt 2009; Arndt et al. 2000; Thong et al. 2006; Han et al. 2002; Schulz et al. 2010). Table 1 lists suitable stimuli-responsive hydrogels for the diverse measurement quantities. It is possible to tailor sensitivity and to decrease non-linearity by using polymer blends or co-polymers.

\subsection{Humidity-and $\mathrm{pH}-$ responsive behaviour} of PVA/PAA

Compounds of PVA und PAA were chosen as a very promising material for humidity-responsive BIZEPSsensors.

- PVA/PAA can easily be processed. Both components (Aldrich Chemical Co.) were separately solved in distilled water at $80{ }^{\circ} \mathrm{C}$ (PVA: 15 wt \%, PAA 7.5 wt $\%$ ) and then mixed with a ratio of $80 \mathrm{wt} \%$ of PVA and $20 \mathrm{wt} \%$ of PAA. After stirring at $60{ }^{\circ} \mathrm{C}$ for $1 \mathrm{~h}$, PVA/PAA blends have been deposited and photo crosslinked $\left(130{ }^{\circ} \mathrm{C}, 20 \mathrm{~min}\right)$.

Table 1 Stimulus-responsive hydrogels (Guenther 2009; Guenther et al. 2009, 2010; Schulz et al. 2009; Miyata et al. 1999)

\begin{tabular}{ll}
\hline $\begin{array}{l}\text { Stimulus, } \\
\text { measurement quantity }\end{array}$ & Polymer \\
\hline $\begin{array}{l}\text { Relative humidity } \\
\text { pH }\end{array}$ & PVA/PAA \\
& PVA/PAA \\
& DMAEMA-co-HEMA \\
& PMAA-PEGDMA-DMPA \\
Temperature & PNIPAAm(BIS4) \\
Concentration of & P2VP-block-P(NIPAAm-co-DMIAAm) \\
heavy metal ions & P4VP-block-P(NIPAAm-co-DMIAAm) \\
Salt concentration & PNIPAAm(BIS4) \\
(NaCl, NaJ) & P2VP-block-P(NIPAAm-co-DMIAAm) \\
& PNIPAAm-DMAAm-DMIAAm \\
Ethanol concentration & PNIPAAm(MBAAm4) \\
Glucose & AAm/3-APB \\
Protein concentration & P4VP-block-P(NIPAAm-co-DMIAAm) \\
(human serum & \\
albumin, HSA) & \\
\hline
\end{tabular}


- PVA/PAA shows good long-term stable behaviour (Guenther 2009).

- The volume extension of the hydrogel creates a larger bending of the flexure plate then that one of bimorphs. Therefore, the arrangement of Fig. 3 using volume extension instead of a swelling bimorph layer seems to be advantageous and will be used in the following.

To match the stiffness of the bending plate and the mechanical properties of the hydrogel to achieve a maximum sensitivity the elastic parameters of PVA/PAA was studied as a function of relative humidity (Fig. 4). The experiments were performed using a tensile testing machine.

The results show that the maximum strain is $7 \%$ at a maximum relative humidity of $100 \%$. However, the Young's modulus decreases sharply at higher relative humidity values. Because the preferred application of humidity switches will be in the higher $\mathrm{RH}$ range the thickness of the polymer has to be chosen according to the thickness of the silicon bending plate. This underlines the superiority of the approach using the volume effect instead of the bimorph type sensor.

The time-dependant swelling behaviour of PVA/PAA was previously for the example of $\mathrm{pH}$ value changes in aqueous solutions (Fig. 5). The swelling kinetics is determined by cooperative diffusion processes for the chemical species (Gerlach and Arndt 2009). Therefore, it is proportional to the square of the characteristic length molecules or ions have to travel. The larger the hydrogel volume is the longer the diffusion process will be. Hence, the optimum hydrogel dimensions has to be a trade-off between fast sensor response and high sensitivity. Very short sensor response times in the range of a few seconds are only possible if the hydrogel is used as a thin film. Thicknesses of a few $100 \mu \mathrm{m}$ lead to characteristic time constants in the range of minutes.

Figure 5 also shows that the diffusion process might comprise several mechanisms (Guenther et al. 2007). After the $\mathrm{pH}$ value jumps an instantaneous volume change takes place due to the hydrogel's elastic behaviour (phase 1).

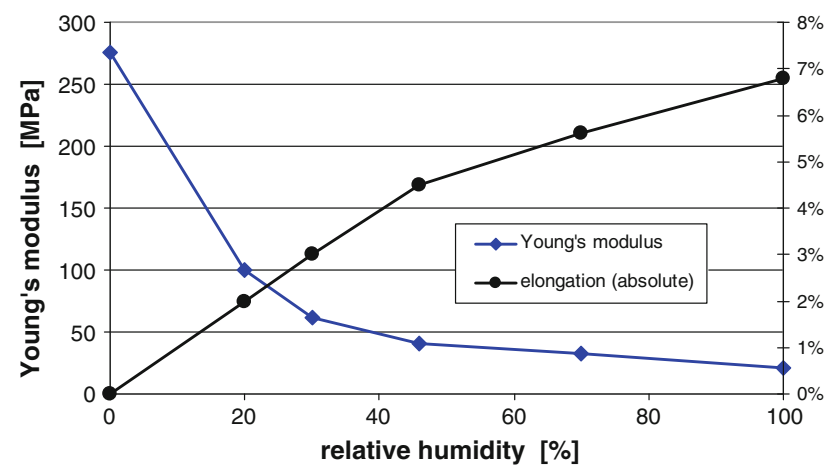

Fig. 4 Young's modulus and relative elongation (strain) versus relative humidity $\left(24{ }^{\circ} \mathrm{C}\right)$

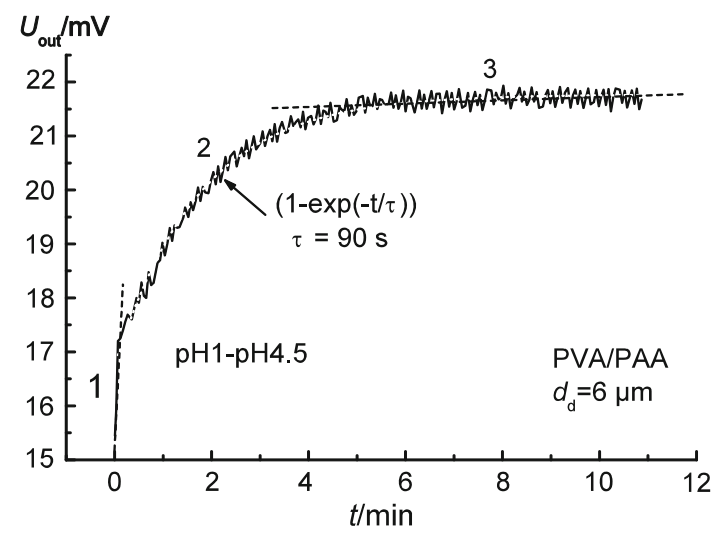

Fig. 5 Sensor output voltage during the swelling process of PVA/PAA from $\mathrm{pH} 1$ to $\mathrm{pH} 4$

The following exponential response (phase 2) is caused by the above-mentioned cooperative diffusion. In the case of polyelectrolyte polymers in connection with ion flux, unwanted drifts might occur (phase 3 ). This was observed for PVA/PAA under the influence of $\mathrm{H}^{+}$-ions as it happens in $\mathrm{pH}$ sensors. The application of PVA/PAA for humidity sensor switches excludes these ions and, hence, avoids this drift behaviour.

\section{Transducer}

The conversion of the movement into a switching operation and a contact force is the second important component of the BIZEPS sensor because it establishes the connection between the identification system and the contacts. A serious problem concerning mechanical switching is the dependence of the contact resistance on the contact force. The contact force should amount to a certain given value because the contact resistance decreases with increasing contact force due to Hertzian stress and the resulting larger cross section of the contact. Due to mechanical boundary conditions and dynamic reasons, the contact resistance cannot switch infinitely fast from "open" $(\mathrm{R} \rightarrow \infty)$ to "closed" $(\mathrm{R} \rightarrow 0)$ and vice versa. If the transit time is not short enough the contact force facilitates the formation of electric arcs, depending on the load to be switched, and therefore causes an increased power dissipation in the moment of switching which might damage the contacts. Conventional mechanical switches avoid this state by a sudden switching process as a result of hysteresis-related switching characteristics. This can be achieved if the mechanical energy, stored in the pre-stressed bending plate, is released abruptly at the transition point. For this reason, usually bi-stable mechanisms are implemented (Seki et al. 1997; Qui et al. 2003).

Most commonly, flexure beams and flexure plates are used as basic mechanical elements. However, flexure plates are to prefer because they separate the measurand (in this 
case relative humidity) hermetically from the electronic components including switching contacts and conductors. By doing so, this facilitates the integration process of the functional groups enormously and ensures compatibility.

In our experiments we used commercial silicon pressure sensor chips processed with semiconductor and MEMS technologies. The size of the chips amounts to $3 \times 3$ to $5 \times 5 \mathrm{~mm}^{2}$ with a 5-20 $\mu \mathrm{m}$ thin membrane (flexure plate) of a size of $1 \times 1$ to $3 \times 3 \mathrm{~mm}^{2}$. Polymer and hydrogel films are either deposited onto the membrane by spincoating or placed into the cavity as pre-casted pieces or are filled into the cavity as solution with subsequent chemical polymerization.

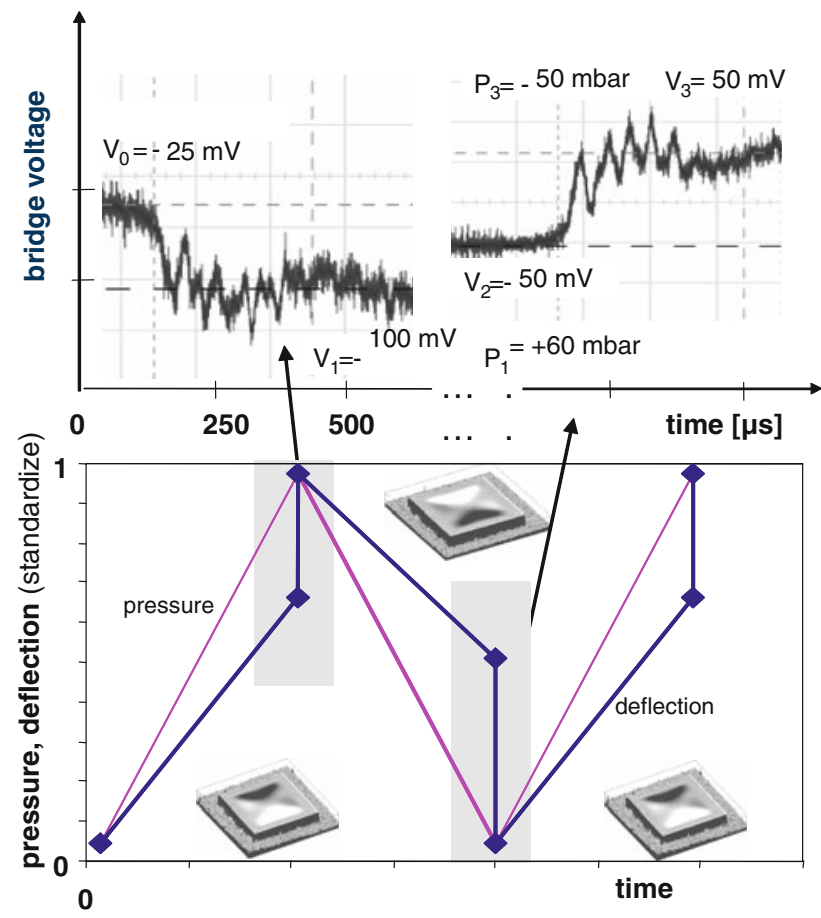

Fig. 6 a Switching kinetics of a pre-stressed bending plate for a continuous pressure change up to +60 mbar and back to -50 mbar, b normalized pressure curve and corresponding normalized deflection of the bending plate, measured using integrated piezoresistive resistors in the bending plate (square bending plate area $9 \mathrm{~mm}^{2}$, thickness $20 \mu \mathrm{m}$ )
Figure 6 shows the bi-stable switching behavior of a prestressed flexure plate under the influence of an applied pressure. In these experiments pressure was used to mimic the volume swelling and shrinkage of the hydrogel. The measurement of the switching kinetics was performed using square plates with integrated piezoresistive Wheatstone bridges. This allows the direct measurement of both the plate movement and the mechanical stresses within the plate.

When the flexure plate is loaded from the bottom with a surface load and the load is increased continuously, the plate snaps into the other at about $60 \mathrm{hPa}$. Figure 6 shows that the switching process takes only a few microseconds. At a negative load of $50 \mathrm{hPa}$, the flexure plate snaps back to the initial state.

Figure 7 describes the calculated deflection of a prestressed flexural plate. For this purpose, a simple model based on two coupled, compressed springs was applied which allows both the calculation of the operating force and the contact force as a function of the deflection (Fig. 7a). The results clearly show that pre-stressing the plate is necessary to provide a sufficient contact force to ensure a secure electrical mechanical switching kinetics. Figure $7 \mathrm{~b}$ depicts the calculated pathway versus the operating force when the bias voltage reaches the snapping point. In a next step finite element (FE) calculations will be used to advance the calculation of the sensor behavior.

\section{Conclusions}

The paper introduced the novel concept of BIZEPS as threshold switches which take the switching energy directly from the quantity to be measured. In this manner they deliver the switching states "ON" and "OFF" without any additional electrical energy. The sensor set-up comprises a stimulus-sensitive material which provides the deflection that is transferred into the movement of the switching contact by the transducer element.

As a first example humidity-dependant sensor switches have been considered. PVA/PAA hydrogel was chosen as recognition element, i.e. humidity-measuring component.
Fig. 7 a Mechanical replacement circuit diagram of a pre-stressed bending plate with compression springs, b operating force as the function of the operating path
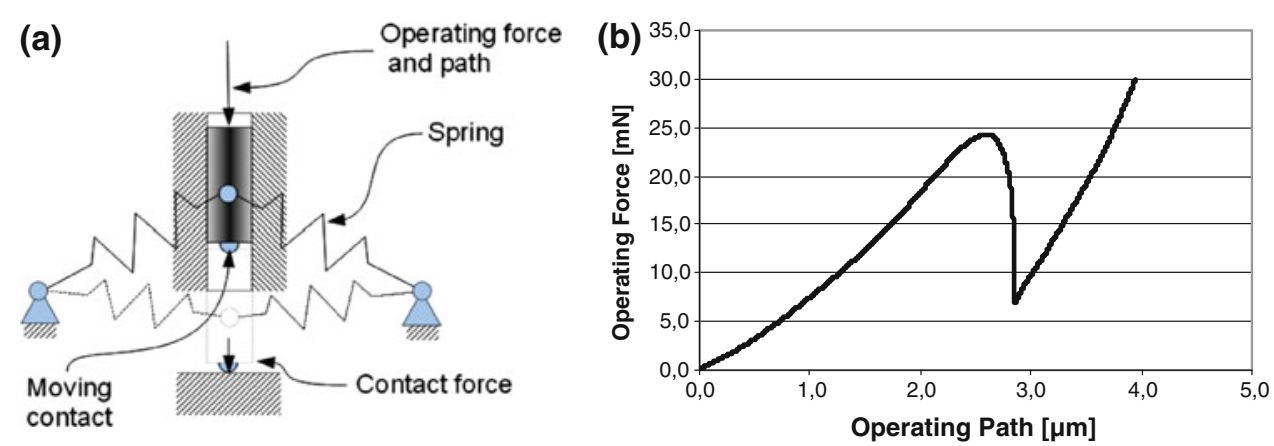
Volume swelling of thick hydrogel elements provides sufficient switching forces. Typical response time constants are in the range of a few minutes. Fast switching within a few microseconds can be achieved using pre-stressed bending plates. This avoids arcing and enables reliable switching. Next steps will be (1) the advancement of calculating the switching behavior by using transient $\mathrm{FE}$ analysis, (2) the investigation of the contact forces to provide low contact resistivity values in the $\mathrm{m} \Omega$-range as well as reliable switching (see for example Hannoe and Hosaka 1996; Bader et al. 2001), (3) the optimization of the sensor set-up, and (4) the study of selected applications.

Open Access This article is distributed under the terms of the Creative Commons Attribution License which permits any use, distribution, and reproduction in any medium, provided the original author(s) and the source are credited.

\section{References}

AMA (2012) AMA Association for Sensor Technology Website. http://www.ama-sensorik.de/site/de/293/umsatzentwicklung.html. Accessed 15 May 2012

Arndt KF, Kuckling D, Richter A (2000) Application of sensitive hydrogels in flow control. Polym Adv Tech 11:496-505

Bader B, Gehrlach F, Scheerer W (2001) Untersuchung des elektronischen Kontaktverhaltens mikromechanischer Schaltelemente. Final report FV-Nr 11687N. Hahn-Schickard-Gesellschaft, Institut für Feinwerk- und Zeitmesstechnik

Beeby SP, Tudor MJ, White NM (2006) Energy harvesting vibration sources for microsystems applications. Meas Sci Technol 17: R175. doi:10.1088/0957-0233/17/12/R01

Buchhold R, Gerlach G (2000) Bimorphe Gassensoren. Techn Mess 67:228-232

Buchhold R, Nakladal A, Büttner U, GerlachG (1998a) The metrological behaviour of bimorphic piezo-resistive humidity sensors. Meas Sci Tech 9:354-359

Buchhold R, Nakladal A, Gerlach G, Sahre K, Eichhorn KJ (1998b) Mechanical stress in microma-chined components caused by humidity-induced in-plane expansion of thin polymer films. Thin Solid Films 312:232-239

Cook-Chennault KA, Thambi N, Sastry AM (2008) Powering MEMS portable devices - a review of non-regenerative and regenerative power supply systems with special emphasis on piezoelectric energy harvesting systems. Smart Mater Struct 17:043001. doi: 10.1088/0964-1726/17/4/043001

Drake J, Jerman H, Lutze B, Stuber M (1995) An electrostatically actuated micro-relay. In: Transducers 95, The 8th International Conference on solid-state sensors and actuators, vol 2, pp 380-383

Eloy JC (2006) Status of the MEMS industry in 2006. Sens Transducers Mag 66:521-525

Gattiglia E, Russel T (1989) Swelling behavior of an aromatic polyimide. J Polymer Sci B 27:2131-2144

Gerlach G, Arndt KF (eds) (2009) Hydrogel sensors and actuators. Springer, Berlin

Gerlach G, Guenther M, Suchaneck G, Sorber J, Arndt KF, Richter A (2004) Application of sensitive hydrogels in chemical and $\mathrm{pH}$ sensors. Macromol Symp 210:403-410

Gerlach G, Guenther M, Sorber J, Suchaneck G, Arndt KF, Richter A (2005) Chemical and $\mathrm{pH}$ sensors based on the swelling behavior of hydrogels. Sens Actuators B 111-112:555-561
Guenther M (2009) Anwendungen polymerer Funktionsschichten in piezoresistiven chemischen und Feuchtesensoren. TUD press, Dresden

Guenther M, Sahre K, Suchaneck G, Gerlach G, Eichhorn KJ (2001) Influence of ion-beam induced chemical and structural modification in polymers on moisture uptake. Surf Coat Technol 142-144:482-488

Guenther M, Sorber J, Gerlach G, Suchaneck G, Thong TQ, Arndt KF, Richter A (2005) Piezoresistive Chemosensoren auf der Basis von Hydrogelen. Techn Mess 72:93-102

Guenther M, Gerlach G, Wallmersperger T (2007) Modeling of nonlinear effects in $\mathrm{pH}$ sensors based on polyelectrolytic hydrogels. In: Electroactive polymer actuators and sevices 2007. Proceedings of SPIE 6524:17-1-11

Guenther M, Gerlach G, Wallmersperger T, Schulz V (2009a) Modeling and experimental investigations of the sensitivity of piezoresistive chemical sensors based on pyroelectric hydrogels. In: Electroactive polymer actuators and devices 2009. Proceedings of SPIE 7287: 0U-1-11

Guenther M, Gerlach G, Wallmersperger T (2010) Piezoresistive biochemical sensors based on hydrogels. Microsyst Technol 16:703-715

Han IS, Han MH, Kim J, Lew S, Lee YJ, Horkay F, Magda JJ (2002) Constant-volume hydrogel osmometer: a new device concept for miniature biosensors. Biomacromol 3:1271-1275

Hannoe S, Hosaka H (1996) Electrical characteristics of micro mechanical contacts. Microsyst Technol 3:31-35

Hei K, Pott V, Nathanael R, Jaeseok J, Alon E, King LiuTJ (2009) Design and reliability of a micro-relay technology for zerostandby-power digital logic applications. In: IEEE International Electron Devices Meeting IEDM 2009, pp 1-4

Miyata T, Asami N, Uragami U (1999) A reversibly antigenresponsive hydrogel. Nature 399:766-769

Ouchi I, Hosoi M, Tomie T (1992) Hygroscopic expansion of polyester films. Jap J Appl Phys 31:2505-2507

Petersen KE (1979) Micromechanical membrane switches on silicon. IBM J Res Dev 23:376-385

Petersen KE (1982) Silicon as a mechanical material. Proc IEEE 70:420-457

Qui J, Lang JH, Slocum AH, Strumpler R (2003) A high-current electrothermal bistable MEMS relay. In: MEMS-03, The 16th Annual International Conference on Micro Electro Mechanical Systems, Kyoto. IEEE, pp 64-67

Rangelow IW, Gerlach G, Bartuch H, Steinke A, Röder R (2009) Micromechanical sensor switch for threshold related switching of electrical contacts, patent WO/2009/077446

Schulz V, Guenther G, Gerlach G, Magda JJ, Tathireddy P, Rieth L, Solzbacher F (2009) In vitro investigation of $\mathrm{pH}$ - and ionicstrength-responsive polyelectrolytic hydrogel using a piezoresistive microsensor. In: Electroactive polymer actuators and devices 2009. Proceedings of SPIE 7287:12-1-10

Schulz V, Gerlach G, Günther M, Magda JJ, Solzbacher F (2010) Piezoresistive $\mathrm{pH}$ microsensors based on stimuli-sensitive polyelectrolyte hydrogels. Techn Mess 77:179-186

Seki T, Sakata M, Nakajima T, Matsumoto M (1997) Thermal buckling actuator for micro relays. In: Transducers 97, The 9th International Conference on solid-state sensors and actuators, vol 2, pp 1153-1156

Suzuki K (2002) Micro electro mechanical systems (MEMS) microswitches for use in DC, RF, and optical applications. Jpn J Appl Phys 41:4335-4339

Thong TQ (2006) Hydrogel-based piezoresistive pH sensors. TUD press, Dresden

Thong TQ, Gerlach G, Sorber J, Arndt KF (2006) Hydrogel-based piezoresistive $\mathrm{pH}$ sensors: design, simulation and output characteristics. Sens Actuators B 117:17-26 